\title{
Altered predictive capability of the brain network EEG model in schizophrenia during cognition
}

\author{
Javier Gomez-Pilar a,*, Jesús Poza ${ }^{\text {a,b,c }}$, Carlos Gómez ${ }^{\text {a }}$, Georg Northoff ${ }^{\mathrm{d}}$, Alba Lubeiro ${ }^{\mathrm{e}}$, Benjamín B. Cea-Cañas ${ }^{\mathrm{e}}$, \\ Vicente Molina $^{\mathrm{c}, \mathrm{e}}$, Roberto Hornero ${ }^{\mathrm{a}, \mathrm{b}, \mathrm{c}}$ \\ a Biomedical Engineering Group, E.T.S. Ingenieros de Telecomunicación, University of Valladolid, Valladolid, Spain \\ b IMUVA, Instituto de Investigación en Matemáticas, University of Valladolid, Valladolid, Spain \\ c INCYL, Instituto de Neurociencias de Castilla y León, University of Salamanca, Salamanca, Spain \\ d Institute of Mental Health Research, University of Ottawa, Ottawa, Canada \\ e Psychiatry Department, University Hospital of Valladolid, Valladolid, Spain
}

\section{A R T I C L E I N F O}

\section{Article history:}

Received 13 November 2017

Received in revised form 10 April 2018

Accepted 29 April 2018

\section{Keywords:}

Modeling;

Neural pathways

Neural synchronization

Schizophrenia

EEG

\begin{abstract}
A B S T R A C T
The study of the mechanisms involved in cognition is of paramount importance for the understanding of the neurobiological substrates in psychiatric disorders. Hence, this research is aimed at exploring the brain network dynamics during a cognitive task. Specifically, we analyze the predictive capability of the pre-stimulus theta activity to ascertain the functional brain dynamics during cognition in both healthy and schizophrenia subjects. Firstly, EEG recordings were acquired during a three-tone oddball task from fifty-one healthy subjects and thirty-five schizophrenia patients. Secondly, phase-based coupling measures were used to generate the time-varying functional network for each subject. Finally, pre-stimulus network connections were iteratively modified according to different models of network reorganization. This adjustment was applied by minimizing the prediction error through recurrent iterations, following the predictive coding approach. Both controls and schizophrenia patients follow a reinforcement of the secondary neural pathways (i.e., pathways between cortical brain regions weakly connected during pre-stimulus) for most of the subjects, though the ratio of controls that exhibited this behavior was statistically significant higher than for patients. These findings suggest that schizophrenia is associated with an impaired ability to modify brain network configuration during cognition. Furthermore, we provide direct evidence that the changes in phase-based brain network parameters from pre-stimulus to cognitive response in the theta band are closely related to the performance in important cognitive domains. Our findings not only contribute to the understanding of healthy brain dynamics, but also shed light on the altered predictive neuronal substrates in schizophrenia.
\end{abstract}

(c) 2018 Elsevier B.V. All rights reserved.

\section{Introduction}

It is well-established that disturbed cognition is a core feature of schizophrenia. Schizophrenia patients often exhibit global IQ deficits (Zanelli et al., 2010) and impairments in several cognitive domains, such as semantic memory (Rossell and Batty, 2008), executive function (Simonsen et al., 2011), and sustained attention (Sánchez-Morla et al., 2009), among others (Sheffield and Barch, 2016; Vöhringer et al., 2013). These impairments are likely related to alterations in prefrontal neural network dynamics in schizophrenia (Mukherjee et al., 2016; Poppe et al., 2016). However, the exact relationship between neural network abnormalities and cognitive impairment remains unclear.

\footnotetext{
* Corresponding author at: Biomedical Engineering Group (GIB), E.T.S. Ingenieros de Telecomunicación, University of Valladolid, Paseo de Belén 15, 47011 Valladolid, Spain. E-mail address: javier.gomez@gib.tel.uva.es (J. Gomez-Pilar).

URL: http://www.gib.tel.uva.es (J. Gomez-Pilar).
}

Cognition has not only been exhaustively studied using a neuropsychiatric approach both in healthy individuals (Leech and Sharp, 2014) and in schizophrenia patients (Moustafa and Gluck, 2011; Vöhringer et al., 2013), but also from a neuroscientific perspective (Li et al., 2016; van den Heuvel and Fornito, 2014). In this context, a dynamical causal model of the brain behavior has been previously proposed (Friston et al., 2003). Despite the number of virtues of the model, dynamical causal modeling requires a high computational cost and the adjustment of several parameters (Thai et al., 2009). Additionally, the complexity of this model makes it rather difficult to draw direct relationship to brain networks without a strong a priori hypothesis. For these reasons, intuitive models focused on explaining the observed neurodynamics, could be helpful. In this regard, the framework of the predictive coding could be the basis to provide a Bayesian inference of the observed environment (Kilner et al., 2007). Predictive coding is based on minimizing prediction error through recurrent interactions among cortical hierarchy levels (Kilner et al., 2007). The neural activity 
encoding a particular brain state determines where the current dynamics are within the hierarchical sequence (Friston and Kiebel, 2009). Therefore, the encoding of a particular state would have a predictive capability of the subsequent state. Perceptual alterations could be then explained by abnormalities in the dynamic mechanisms of predictive coding (Hohwy et al., 2008).

In this study, we propose an intuitive and reliable model of neural network dynamics during a cognitive task, in which the error between the modeled network and the real brain network is recurrently minimized. Thus, the brain network during the pre-stimulus activity (i.e., prior to stimulus presentation or perception) determines the brain network during the subsequent state. It is necessary, therefore, to characterize the brain network in different moments of the task. One approach being considered would be to directly compare these network parameters, i.e. an arithmetic difference, which would summarize the brain dynamics. This approach can be useful to characterize the network changes, but not the underlying neural mechanisms of such changes. A probabilistic model is, therefore, required in order to identify the neural underpinnings associated with the cognitive task. For that purpose, graph-theoretical analyses combined with EEG can be used to provide a mathematical representation of the functional brain network for studying rapid changes in the coordination and synchronization between different regions. Based on previous evidence about the importance of rapid changes in the cognitive processing (Varela et al., 2001), EEG becomes a suitable tool to analyze brain network changes in the range of milliseconds, unreachable by other neuroimaging techniques, such as fMRI. In addition, it is crucial the use of complementary network measures to obtain a comprehensive characterization of the functional brain network (Rubinov and Sporns, 2010). It is generally accepted that functional brain network is well-connected (Power et al., 2013) and complex (Liu et al., 2008). Furthermore, it exhibits an optimal balance between integration and segregation (Deco et al., 2015), as well as between regularity and irregularity (Tononi et al., 1998). Abnormalities in the previously mentioned brain network features have been reported in schizophrenia (Liu et al., 2008; van den Heuvel and Fornito, 2014; Yeo et al., 2016). Therefore, a combination of the previous network characteristics should be helpful to characterize brain network dynamics related to cognition in schizophrenia.

Dysfunctional interactions between brain areas have been repeatedly suggested as a relevant contribution to explain the mental alterations in schizophrenia (Bjorkquist et al., 2016; Friston and Frilh, 1995; Whalley, 2005). Within this framework, disrupted connectivity in long-range interactions plays a central role in this disorder (Dickerson et al., 2010; Friston et al., 2016; Gomez-Pilar et al., 2015; Sigurdsson et al., 2010). It is noteworthy that a relationship between long-range interactions and low frequency bands, such as delta and theta, has been proposed (Uhlhaas and Singer, 2010). Therefore, it is not surprising that noticeable findings have been usually reported in the literature about the strong association between schizophrenia and brain connectivity in the low EEG frequency bands (Ford et al., 2002; Koenig et al., 2001; Uhlhaas and Singer, 2010). Alterations on low frequency bands have been related to a temporal misalignment of working memory function in schizophrenia (Kikuchi et al., 2007). In this regard, it was suggested that the neural activity underlying working memory may be abnormally dominated by slow frequencies in schizophrenia (Northoff and Duncan, 2016). Similarly, theta oscillations were proposed to be the basis for memory integration (Buzsáki, 2005) and topdown processing (Uhlhaas et al., 2008), both impaired in schizophrenia patients (Clare, 1993; Rossell and Batty, 2008). In addition, it has been suggested that cognitive control deficits may contribute to episodic memory deficits in schizophrenia (Barch and Sheffield, 2014), in which hippocampal and prefrontal regions could play an important role. This, jointly with our previous studies (Bachiller et al., 2015; Gomez-Pilar et al., 2018c), lead us to claim the importance of theta band to characterize the dynamical cognitive network. The analysis of the electric brain activity at low frequencies during the performance of an oddball task (related to working memory function and topdown processing) could then enhance our understanding of memory mechanisms in schizophrenia.

In the last decade, several studies assessed the brain network changes during a cognitive task in schizophrenia and healthy individuals, some of them by means of an oddball task (Bachiller et al., 2015; Reijneveld, 2011; Shim et al., 2014). They reported differences in connectivity and/or network features during the cognitive processing. However, for the sake of comparability, it would be appropriate to go a step further and identify a cognitive network model to explain the observed neural dynamics. In a previous study (Gomez-Pilar et al., 2018c), we suggested that network differences between a healthy and a schizophrenia brain could be related with secondary pathways (i.e., pathways between nodes weakly connected) of the brain network during the pre-stimulus activity. These pathways would be strongly reinforced during the cognitive processing, while other connections would remain almost unchanged. These differences could be specifically linked to frequency bands related to memory and hippocampal activity (i.e. low frequency bands).

Hence, the present study aimed at elucidating the dynamical network model during a cognitive task that better fits the brain network changes in a healthy population, as well as the possible abnormalities in schizophrenia. To avoid inter-subject variability, we performed an individualized approach that provides a specific network model for each subject.

\section{Methods and materials}

\subsection{Study subjects}

Thirty-five schizophrenia patients were recruited from the Psychiatry Department at the University Hospital of Valladolid (Spain). Diagnoses were made according to the Diagnostic and Statistical Manual of Mental Disorders, 5th edition (DSM-V) criteria (American Psychiatric Association, 2013). Fifty-one healthy control subjects, keeping a nonstatistically significant age and gender ratio, were also included in the study. Inclusion/exclusion criteria were undertaken identically as in our previous studies (Bachiller et al., 2014; Gomez-Pilar et al., 2018c; Gomez-Pilar et al., 2017) (see Supplementary material for details). Cognitive data were collected using the Spanish version of the Brief Assessment in Cognition in Schizophrenia (BACS) (Segarra et al., 2011). Clinical and sociodemographic characteristics, as well as antipsychotic doses equivalents for patients, are summarized in Table 1 .

All controls and patients gave their informed consent to be included in the study. The study protocol was approved by the research board of the University Hospital of Valladolid (Spain) and was conducted in accordance with the Declaration of Helsinki guidelines.

\subsection{Cognitive EEG task}

All participants performed a three-stimulus oddball task. During the 13-minutes of the auditory oddball paradigm, participants heard binaural tones bursts presented in random series of 600 tones with an interstimulus interval randomly jittered between 1.16 and $1.44 \mathrm{~s}$. Three different tones were presented: target ( $500 \mathrm{~Hz}$-tone; probability; 0.2 ), distractor (1000 Hz-tone; probability; 0.2$)$ and standard $(2000 \mathrm{~Hz}-$ tone; probability; 0.6 ). The participants were asked to keep their eyes closed and to press a button with their right hand whenever they detected the target tones. Only attended target tones were considered for further analyses. The behavioral performance of both groups is included in Table 1. After preprocessing, the number of trials for target condition was $97.41 \pm 9.98$ for controls and $89.26 \pm 17.04$ for patients.

\subsection{EEG network estimation and model reconstruction}

\subsubsection{Acquisition protocol and network analysis}

EEG recordings were acquired at a sampling frequency of $500 \mathrm{~Hz}$ in 28 electrodes with a BrainVision ${ }^{\circledR}$ equipment (Brain Products $\mathrm{GmbH}$; 
Table 1

Sociodemographic and clinical characteristics of schizophrenia patients and healthy control subjects.

\begin{tabular}{|c|c|c|c|c|c|c|c|}
\hline & & \multicolumn{2}{|c|}{ Controls } & \multirow{2}{*}{$\frac{\text { Patients }}{\text { Mean }}$} & \multicolumn{3}{|c|}{ Comparison } \\
\hline & & Mean & SD & & SD & $t$ & $p$ \\
\hline \multirow[t]{2}{*}{ Demographic data } & Age (years) & 29.31 & 9.74 & 32.68 & 10.37 & -1.510 & 0.135 \\
\hline & Gender (male:female) & $23: 28$ & & $20: 15$ & & $\chi^{2}=0.851$ & 0.356 \\
\hline \multirow[t]{3}{*}{ Symptom scale scores } & PANSS + & - & - & 12.63 & 7.53 & - & - \\
\hline & PANSS - & - & - & 18.26 & 8.24 & - & - \\
\hline & PANSS total & - & - & 54 & 21.47 & - & - \\
\hline \multirow[t]{6}{*}{ BACS scale } & Working memory & 20.67 & 4.00 & 15.79 & 5.31 & 4.601 & $<0.001$ \\
\hline & Processing speed & 70.00 & 14.10 & 42.45 & 15.42 & 8.055 & $<0.001$ \\
\hline & Executive function & 17.18 & 2.63 & 15.57 & 3.44 & 2.311 & $<0.05$ \\
\hline & Verbal memory & 51.61 & 8.57 & 34.76 & 11.25 & 7.457 & $<0.001$ \\
\hline & Motor speed & 72.16 & 14.11 & 47.34 & 14.69 & 4.415 & $<0.001$ \\
\hline & Verbal fluently & 27.89 & 5.77 & 17.44 & 6.39 & 7.103 & $<0.001$ \\
\hline \multirow{2}{*}{ Illness } & Drug equivalence $(\mathrm{mg} / \mathrm{d})$ & - & - & 351.29 & 270.10 & - & - \\
\hline & Duration (months) & - & - & 84.45 & 117.40 & - & - \\
\hline \multirow[t]{4}{*}{ Oddball task } & Reaction time (ms) & 242.43 & 33.06 & 277.23 & 47.32 & -4.018 & $<0.001$ \\
\hline & Precision (\%) & 98.70 & 2.13 & 89.82 & 16.11 & 3.897 & $<0.001$ \\
\hline & Amplitude $\mathrm{Pz}(\mu \mathrm{V})$ & 3.35 & 1.48 & 2.29 & 0.95 & 3.725 & $<0.001$ \\
\hline & Latency Pz (ms) & 448.78 & 86.22 & 457.49 & 117.11 & -0.397 & $>0.05$ \\
\hline
\end{tabular}

PANSS: Positive and Negative Syndrome Scale.

BACS: Brief Assessment in Cognition in Schizophrenia.

Munich, Germany) while the participants underwent the previously mentioned oddball task. Electrode impedance was always kept under $5 \mathrm{k} \Omega$ and each channel was referenced over Cz electrode. After a preprocessing to reduce the noise in the EEG recordings (see Supplementary material for details), brain networks were estimated.

The connectivity values of the functional brain network were computed using the phase-locking value (PLV) across successive trials in the theta frequency band $(4-8 \mathrm{~Hz})$. Once the connectivity matrices were obtained, five complementary network features were assessed: (i) integration, (ii) segregation, (iii) connectivity strength, (iv) complexity, and (v) irregularity. They were quantified by means of the characteristic path length, the clustering coefficient, the graph density, the Shannon graph complexity and the Shannon graph entropy, respectively. Since the last two measures have been recently introduced, they do not have widespread use. In summary, the graph irregularity of the brain network was characterized by the Shannon Graph Entropy, defined as follows (Gomez-Pilar et al., 2018c):

$H=\frac{-1}{\log _{2} T} \sum_{i=1}^{N} \sum_{j>i} \frac{w_{i j}}{W} \log _{2} \frac{w_{i j}}{W}$,

where $W$ is the sum of all weights of the graph and $\log _{2} T$ is a normalization factor introduced to ensure that $0 \leq H \leq 1$. On the other hand, graph complexity was estimated using the Shannon Graph Complexity, defined as follows (Gomez-Pilar et al., 2018c):

$S G C=H \cdot \sqrt{\frac{1}{T-1}} \cdot \frac{\sigma}{\bar{w}}$

where $\bar{w}$ is the average of all edge values of the graph and $\sigma$ is the standard deviation of those values. More details about network matrices generation and network parameter definitions have been included in the Supplementary material.

To evaluate brain network changes during the cognitive task, network measures were computed during the pre-stimulus of each trial (i.e., time interval ranging from $300 \mathrm{~ms}$ before to the stimulus onset) and during the subsequent brain response (from the stimulus onset to $700 \mathrm{~ms}$ after it), with special attention on the brain response related to P3 potential (i.e., a time window of $300 \mathrm{~ms}$ centered on $300 \mathrm{~ms}$ ) (Gomez-Pilar et al., 2017). This procedure is also useful to avoid confounding factors due to volume conduction effects (Bastos and Schoffelen, 2016).

\subsubsection{Dyanmical network modeling during cognition}

The dynamical network model was individually identified for each subject. We considered six different models of brain dynamics. Among all possible models, the six models explained below were selected for being intuitive and easy to explain in physiological terms. As we will discuss later, we are aware that changes in the brain network are probably more complex. The considered models are the following:

i) Reinforcement of primary connections. This model assumes that the primary connections of the brain (i.e. connections with higher values of connectivity measured by PLV) during pre-stimulus will suffer more marked changes during the cognition. Specifically, the connection values are increased during the cognitive processing.

ii) Reinforcement of secondary connections. This model assumes that the secondary connections of the brain (i.e. connections with lower values of connectivity measured by PLV) during pre-stimulus will suffer a more marked increase during cognition.

iii) Reinforcement of a connection at random. This model assumes that the increase of the brain connections during the pre-stimulus can randomly occur.

We also took into account three additional models, which are similar to models i), ii) and iii) but considering a decrease in the edge values:

iv) weakening of primary connections,

v) weakening of the secondary connections and

vi) weakening of a connection at random.

In order to determine the dynamical network model for each subject, an iterative algorithm was used. The schematic overview of the procedure is shown in Fig. 1. In summary, the algorithm modified the connections of the pre-stimulus activity following the models previously described. After repeating the algorithm for each of the six models, the model with the lowest mean square error (MSE) between the real and the modeled response was selected. It ensures that the selected model is the one that better fits the cognitive response. All the steps of the algorithm are detailed in the Supplementary material. 


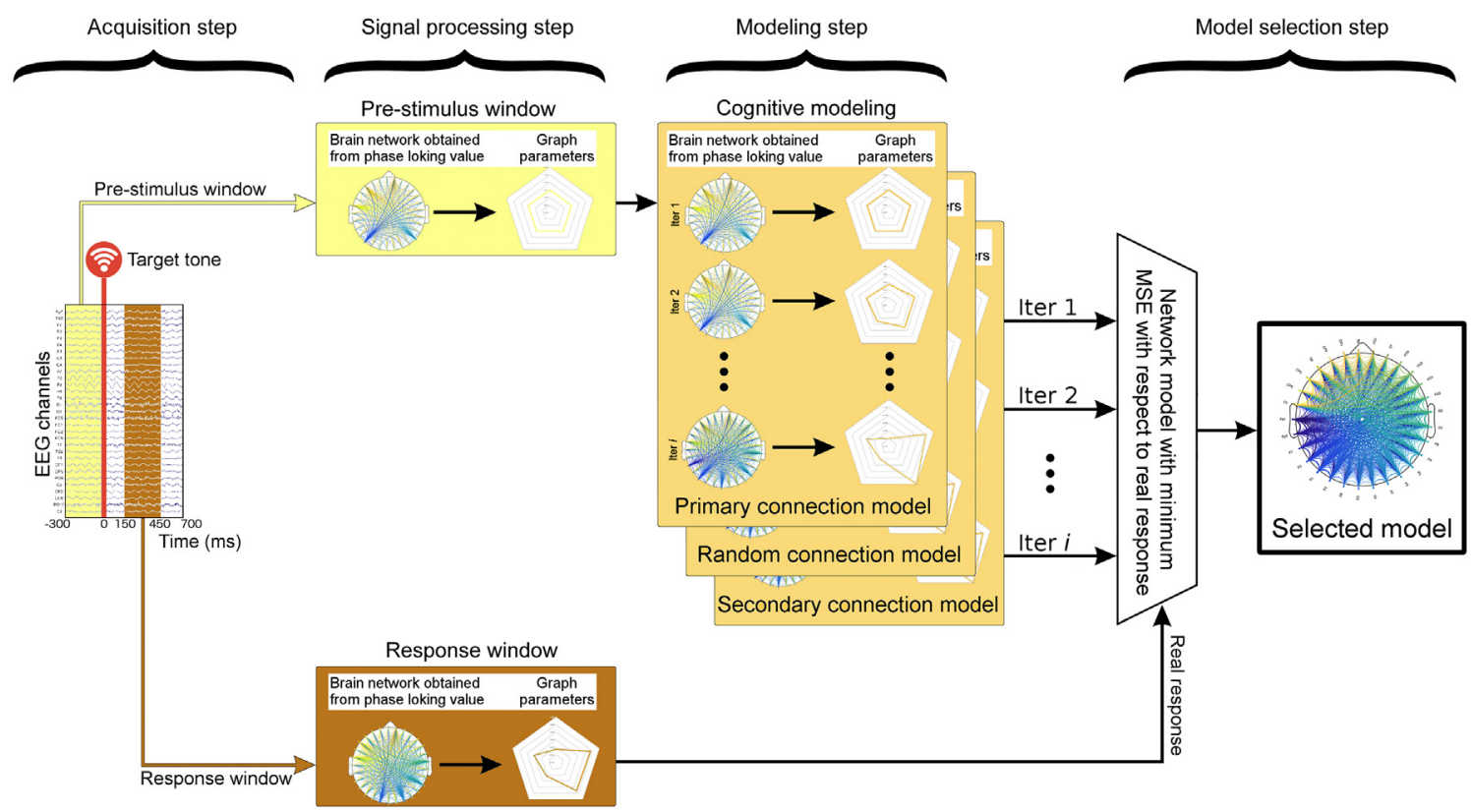

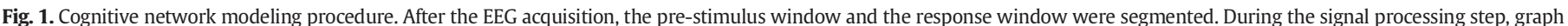

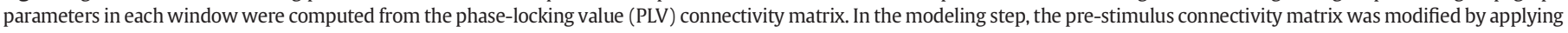

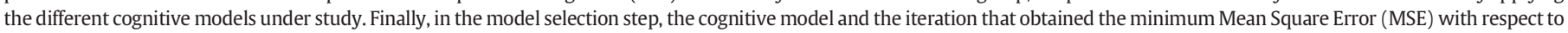
the network parameters in the response window were selected.

\subsection{Statistical analysis}

Statistical analysis was done with SPSS (version 19) and Matlab 'Statistics and Machine Learning' Toolbox (version 2013b). After checking that parametric assumptions were not meet, group differences in gender and age distribution were tested using the Chi-squared test and the Mann-Whitney $U$ test, respectively. Network measures showed a non-Gaussian distribution. Thus, depending on the number of groups, between-group differences were tested using the Mann-Whitney $U$ test or Kruskal-Wallis $H$ test. The effects of age, gender and psychoactive drugs on PLV, network measures and cognitive data were assessed using Spearman's bivariate correlation test (see "Confounding factors" of the Supplementary material for further details), which is robust against spurious since it deals with monotonic associations in a flexible manner. This test was also used for correlation analyses between graph parameters, cognition and symptoms. Finally, Chi-squared test was used for the between-group comparison of the model distributions.

For all the tests, a significance level of $\alpha=0.05$ was used. The Bonferroni correction was applied to control the multiple comparisons problem in the correlation analyses between network parameters and cognition. No correction for multiple comparison was performed when comparing graph parameters, since measures were obtained at network level, i.e. one value for each network.

\section{Results}

\subsection{Network dynamics}

A visual comparison of the averaged brain networks before and after the stimulus onset (see Fig. 2) shows a global increase of the edge weight values for both groups, though this increase is more noticeable for controls. The brain networks were visualized using the BrainNet Viewer (Xia et al., 2013). To assess network evolution across time, a sliding window approach was used. Windows of $300 \mathrm{~ms}$ with an overlap of $90 \%$ were selected for network measures computation. Fig. 3A shows the associated dynamics for each network parameter and group. Statistically significant between-group differences for each time window were marked with black rectangles. The main differences were found around the N2 and $\mathrm{P} 3$ event-related potentials.

Fig. 3B depicts violin plots with the distribution of the averaged change of the network parameters from pre-stimulus to cognitive response for both groups. Statistically significant differences between groups for the change from pre-stimulus activity to cognitive response were obtained: integration $(U(84)=619$; $p<0.05)$, segregation $(U(84)=553.0 ; p<0.01)$, connectivity strength $(U(84)=$ $559.5 ; p<0.01)$, complexity $(U(84)=670.5 ; p<0.05)$ and irregularity $(U(84)=670.5 ; p<0.05)$. In summary, brain network during prestimulus window has lower changes in the response window in schizophrenia patients compared to healthy controls.

\subsection{Modeling the network changes}

Network modeling with the three different scenarios (primary, secondary and random connection models) was applied to data obtained from the pre-stimulus window. Thus, the model that better predicts the cognitive response network using the pre-stimulus network was selected individually for each subject. The behavior of the modeling is shown in Fig. 4. On average, the MSE between the network parameters in pre-stimulus window and the response window was $10.69 \%$ for controls and $2.96 \%$ for patients (from yellow to orange lines). Furthermore, the MSE between the predicted model and the cognitive response was $0.21 \%$ for controls and $0.07 \%$ for patients, which serves to exemplify the accuracy of the model.

The procedure selected a single model for each subject. The distribution of the model for each group is shown in Fig. 5A. Secondary reinforcement model was selected for most of the subjects, especially in the control group. Models based on weakening network connections were selected only in $15 \%$ of the subjects (thirteen subjects: seven controls and six schizophrenia patients; more details are indicated in the Supplementary material). Statistically significant differences between groups in the model selection distribution were obtained $\left(\chi^{2}(2, N=73)=6.6874, p<0.05\right.$; Chi-square test $)$. Likewise, MSE distribution of the network parameters in each cognitive model was also assessed for both groups (Fig. 5B). Within-group comparisons indicate that controls exhibited a statistically significant different MSE 
Pre-stimulus window

\section{Healthy controls}

\section{Schizophrenia patients}

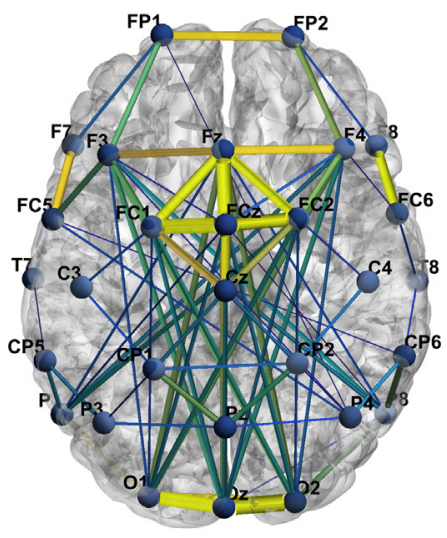

\section{Response window}
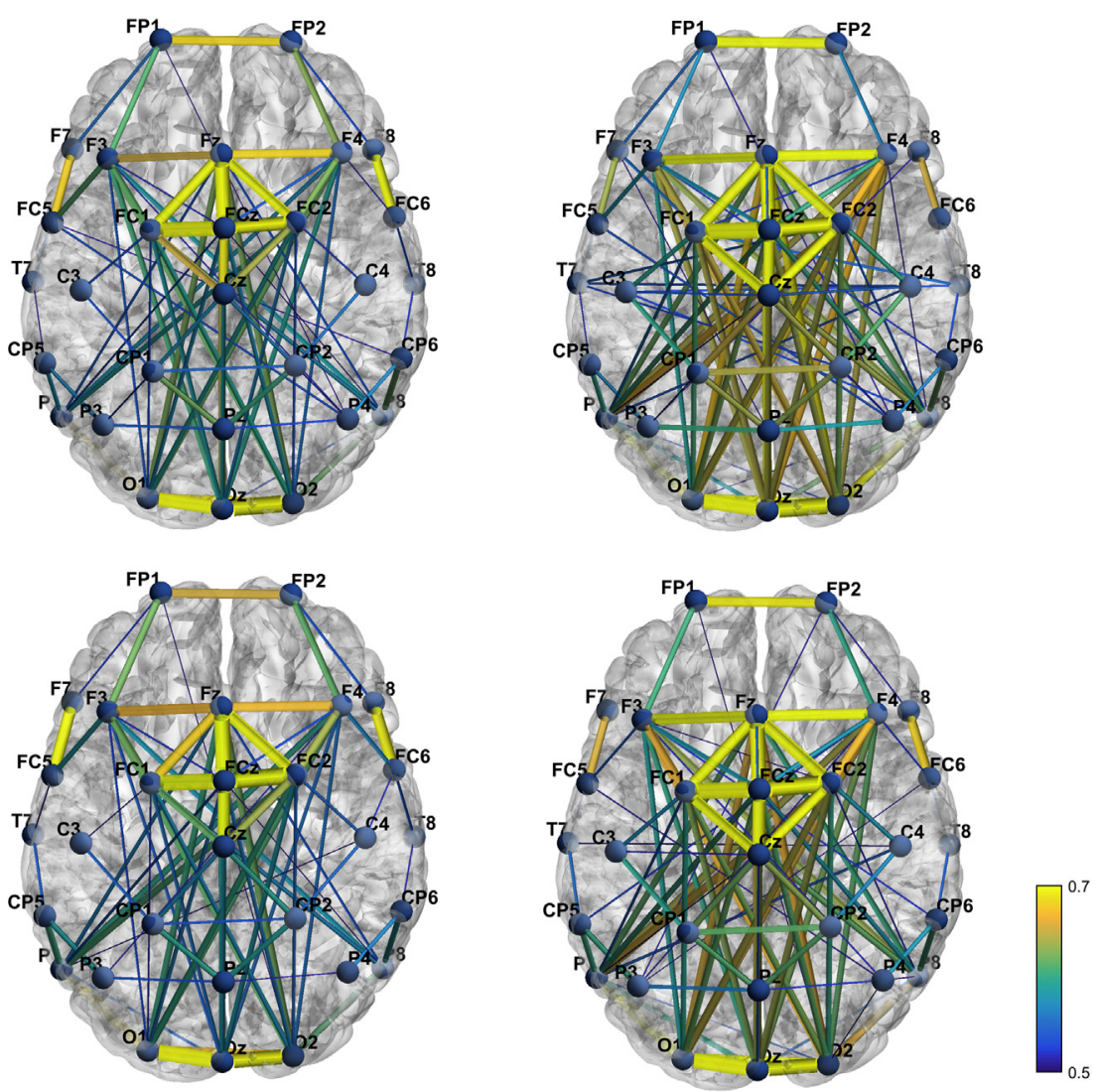

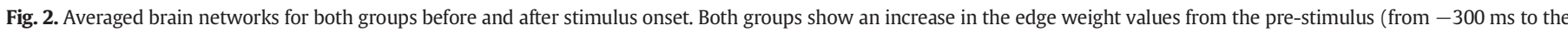

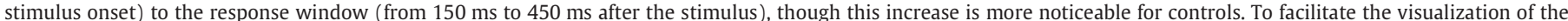

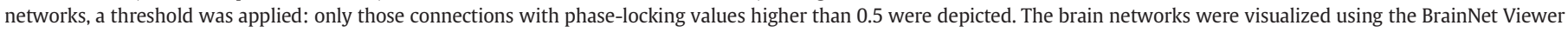
(Xia et al., 2013).

distribution $\left(\chi^{2}(2, N=44)=22.0032, p<0.001\right)$, but not schizophrenia patients $\left(\chi^{2}(2, N=29)=2.6302, p>0.05\right)$. In summary, the higher change from pre-stimulus activity to cognitive response for controls, the higher probability to model their behavior by a reinforcement of the secondary connections. The schizophrenia patients do not follow this tendency, as they do not exhibit a relevant change from pre-stimulus activity to the post-stimulus period during the cognitive response.

\subsection{Cognitive correlates}

Interest in the assessment of correlations between cognition and brain functioning is growing, as it is becoming increasingly evident that it is a useful way to evaluate the heterogeneity of schizophrenia (Sheffield and Barch, 2016). As shown in Fig. 6, there was a noticeable relationship between connectivity strength modulation (measured as the percent of change from pre-stimulus to cognitive response) and cognitive parameters ( $z$-score corrected).

Specifically, the connectivity strength was statistically significant correlated to processing speed $(r=0.472, p<0.001)$, verbal fluency $(r=0.499, p<0.001)$ and verbal memory $(r=0.423, p<0.001)$ after Bonferroni correction. The positive correlations indicate that the greater susceptibility to change in the pre-stimulus, the better cognitive performance. No other statistically significant correlations were found after Bonferroni correction.

Additionally, we performed correlations between cognition and symptoms, as well as between network parameters and symptoms. All these correlations are shown in the Supplementary material.

\section{Discussion}

To the best of our knowledge, this is the first study that combine network modeling and EEG recordings to determine a model of network dynamics during cognition for healthy and schizophrenia subjects. The proposed network modeling effectively predicts the functional brain network of the cognitive response from the pre-stimulus activity.

\subsection{Disrupted brain dynamics of the phase-based mechanisms in schizophrenia}

Schizophrenia has been characterized by abnormal brain network reconfigurations, commonly described in the literature (Gomez-Pilar et al., 2017; Shim et al., 2014). These abnormal dynamics (see Fig. 3) suggest a disrupted phase-based mechanisms during the cognitive processing (Lakatos et al., 2013).

In this study, a significant reduced dynamic capability of the network during the pre-stimulus was observed in patients. This reduction is driven by the phase of the EEG theta band. The lack of change in schizophrenia patients was characterized by complementary network parameters. All of them showed statistically significant reduced changes, which involves an impaired ability to modify the main topological features of the brain network. The reduced flexibility of the network integration and connectivity strength during the task supports an impaired capability of the communication among brain network, which is in agreement with the results obtained by previous studies (Bob et al., 2008; Friston, 1998; Kim et al., 2003). The lower change on segregation in functional brain networks indicates lower local 
A
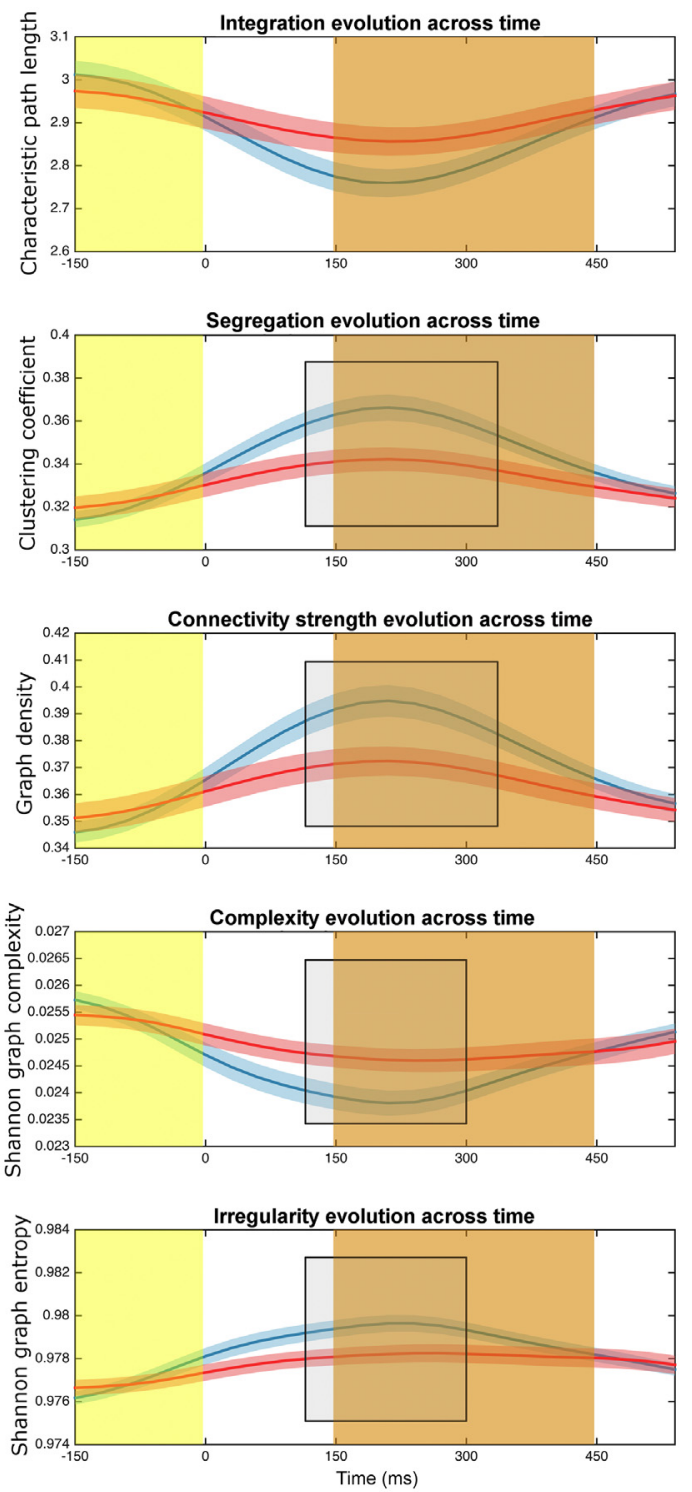

B
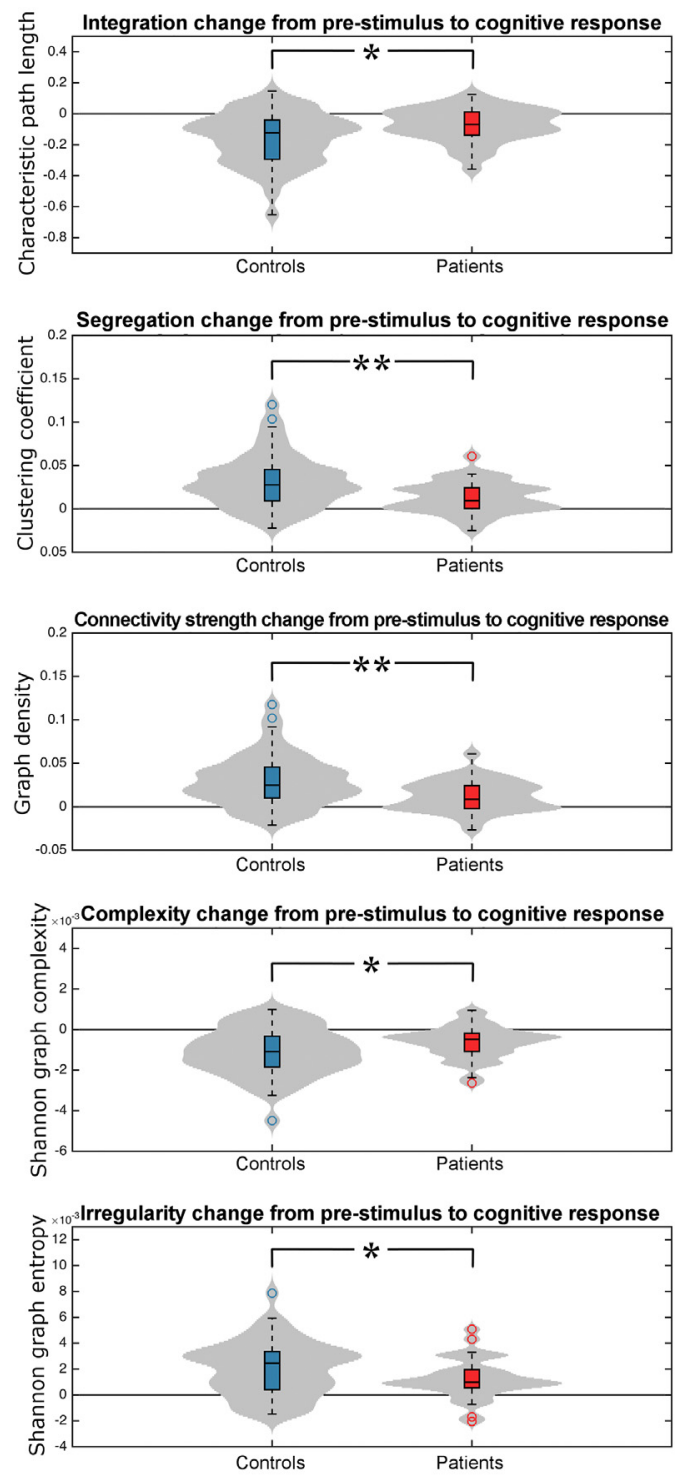

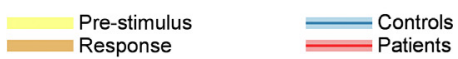

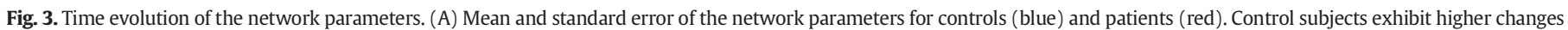

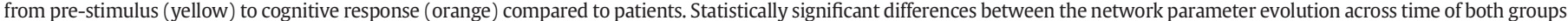

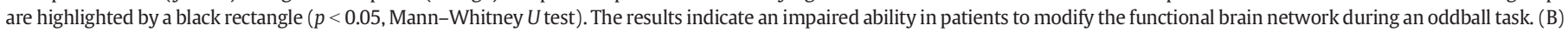

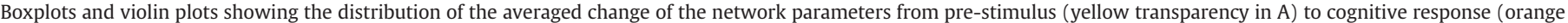

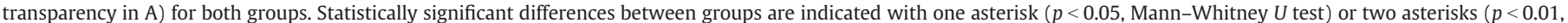
Mann-Whitney $U$ test).

communication or less segregated neural processing (Rubinov and Sporns, 2010). Finally, a reduced change in graph regularity and graph complexity was also found for patients. The physiological interpretation of this result could be related to abnormalities in small-world structure (Liu et al., 2008; Micheloyannis et al., 2006) and, therefore, a reduced network efficiency (Bassett and Bullmore, 2006; Boccaletti et al., 2006).

The underlying biological mechanisms that influence the abnormal dynamics can have different explanations. From the graph theory point of view, a fMRI study reported a diminished number of hubs during a cognitive task in schizophrenia (Ma et al., 2012). These wellconnected nodes typically increase the global integration and connectivity, as well as local segregation. Therefore, the lower the number of hubs in schizophrenia during a cognitive task, the lower the global integration, connectivity and segregation. From a physiological perspective, this lack of change can be related to the abnormal oscillatory behavior during a cognitive task in schizophrenia, which could elicit a lower synchronization between brain regions in comparison to healthy subjects. It could be explained by an abnormal balance between inhibitory GABAergic interneurons (Lewis et al., 2005; Moghaddam, 2003) and pyramidal neurons producing dysfunctionalities between excitation and inhibition processes, which is reflected in the phase measures. This affects to the neural pathways in long-range synchronization (Dickerson et al., 2010), providing abnormal phase-based network measures. Hence, the result that the diminished EEG response was observable in complementary network parameters (see Figs. 3 and 4) suggests that different brain network domains are significantly affected by schizophrenia. 


\section{Controls}

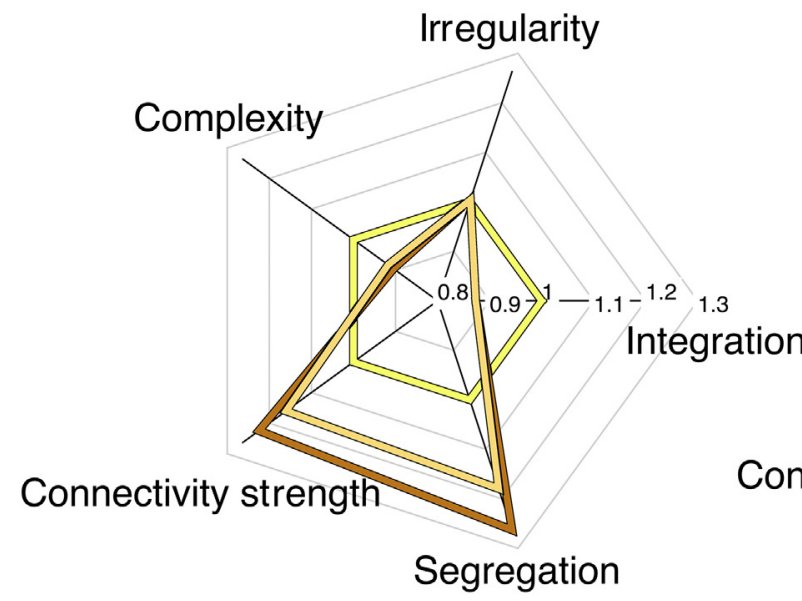

Patients

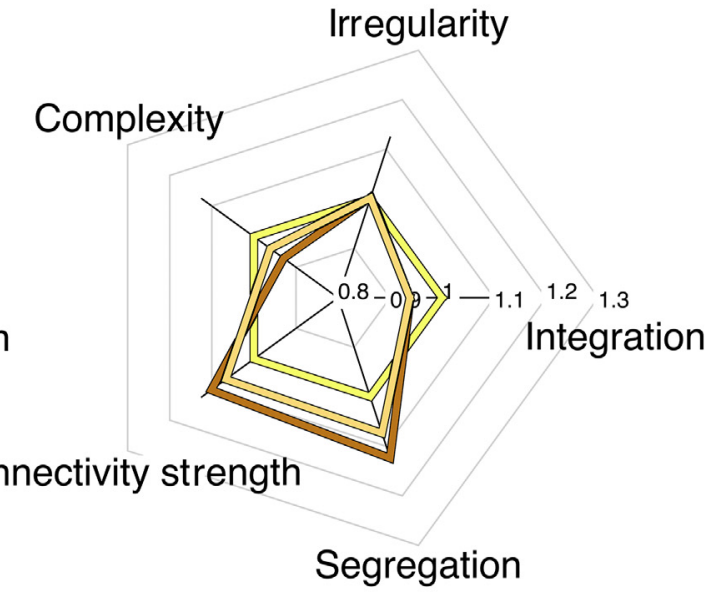

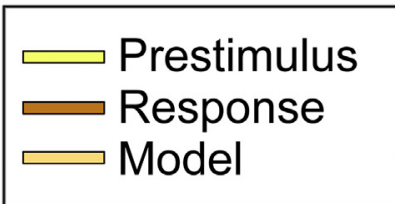

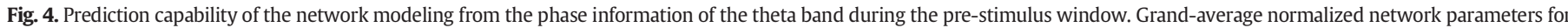

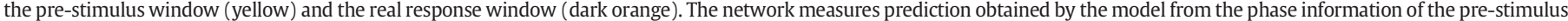
window is also shown (light orange). The model fitting for both the controls and the patient groups is computed by minimizing the mean square error (MSE).

\subsection{Reinforcement of the secondary pathways as a predominant model}

The results show (Fig. 5A) that the predominant model of brain network dynamics during cognition in healthy and schizophrenia subjects involves a reinforcement of the secondary pathways of the pre-stimulus network (i.e., connections with lower phase synchronization between brain areas prior to stimulus perception). However, there are statistically significant differences in the model distribution between groups.
In order to provide a reason of these differences, we rely on the predictive coding (Hohwy et al., 2008). In this study, we constructed generative models that minimize the error at each iteration following the main neurocomputational principle for the brain perception of the environment (Hohwy et al., 2008). The recurrent error minimization until the most likely model has been obtained is formally equivalent to empirical Bayesian inference (Kilner et al., 2007). This implies that abnormalities in the dynamical process of the brain reconfiguration would
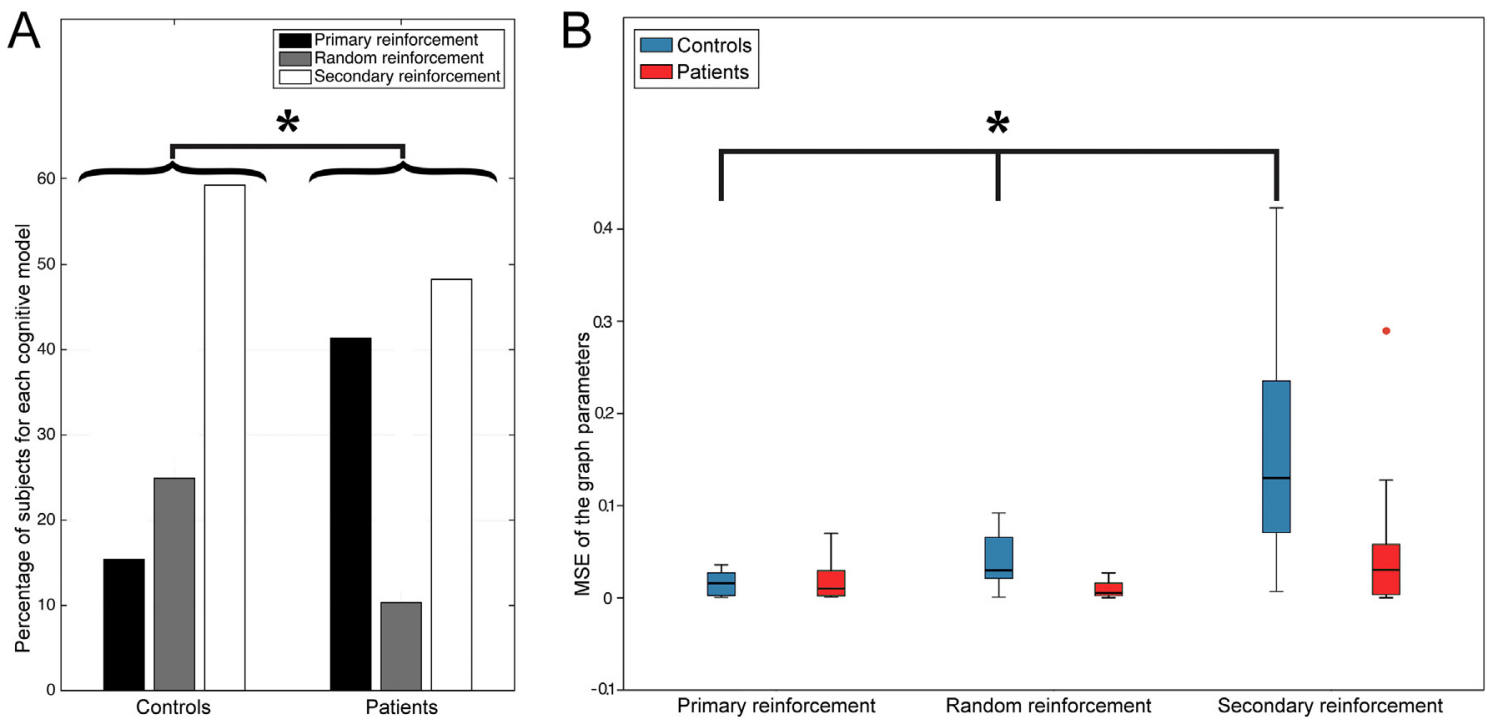

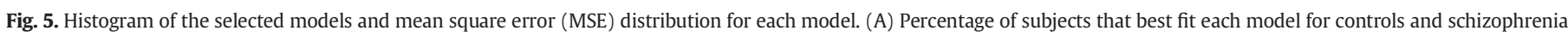

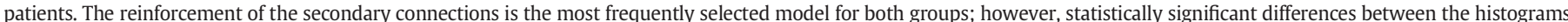

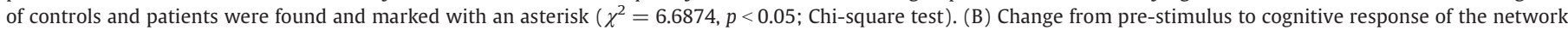

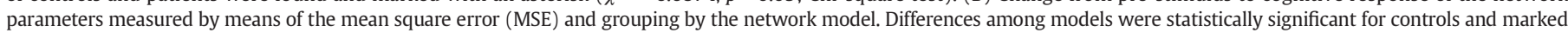
with an asterisk $\left(\chi^{2}(2, N=73)=22.0032, p<0.001\right.$; Kruskal-Wallis test $)$, but not for patients $\left(\chi^{2}(2, N=73)=2.6302, p>0.05\right.$; Kruskal-Wallis test $)$. 

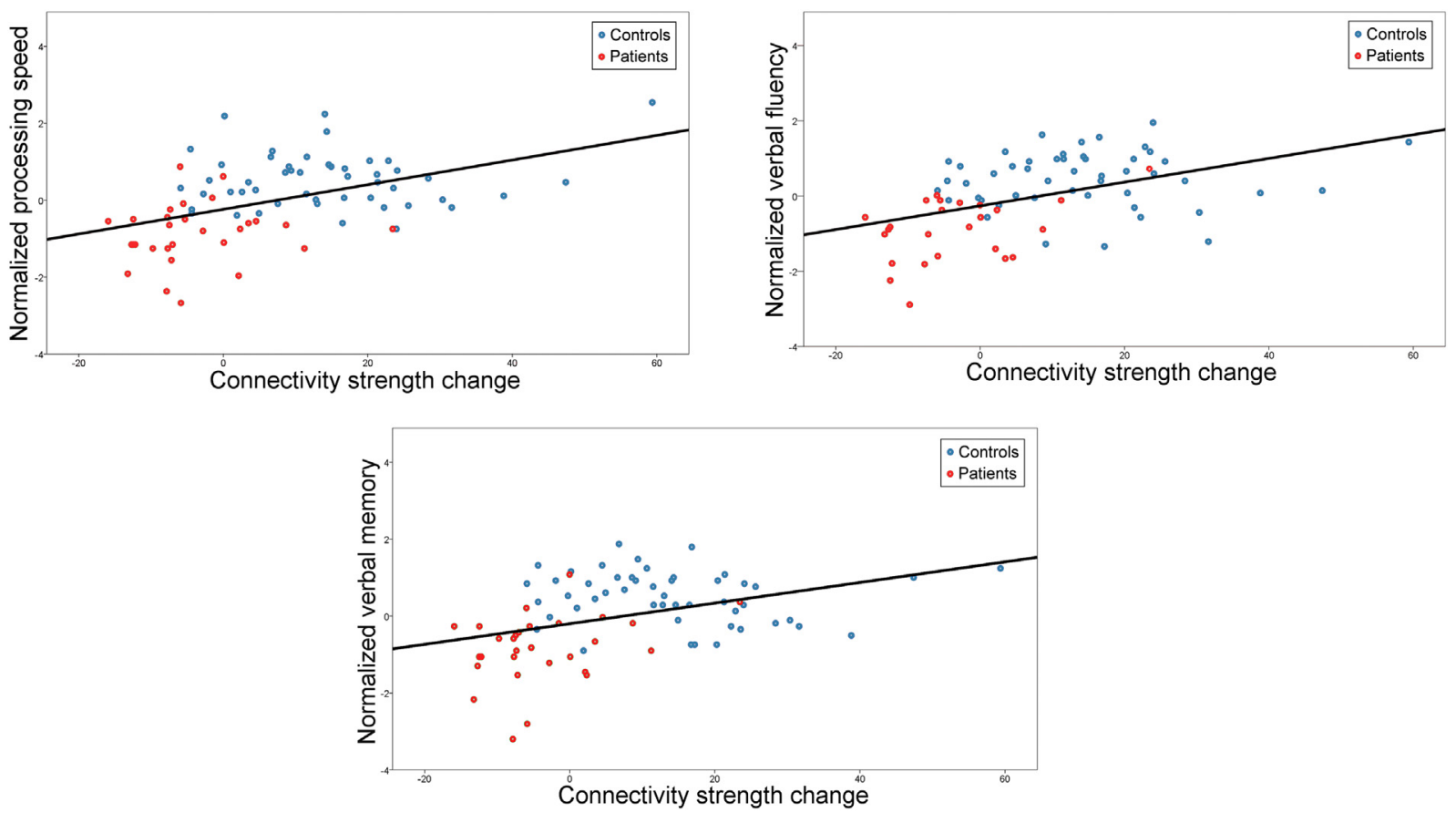

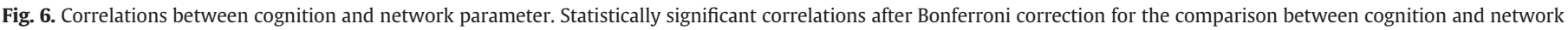

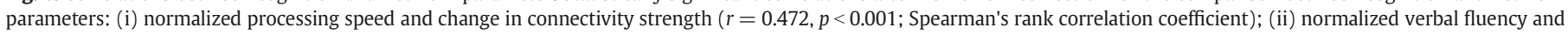

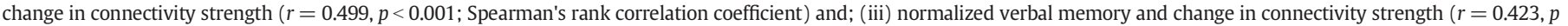
$<0.001$; Spearman's rank correlation coefficient).

have a strong impact on the subsequent state. Following the aberrant salience hypothesis (Kapur, 2003), schizophrenia leads to an aberrant assignment of salience to the elements of one's experience. It suggests that the relevance assignment to the unexpected stimulus in an oddball task would be disrupted in schizophrenia and, as a consequence, would cause an increase in the prediction error. Therefore, the observed disrupted brain dynamics in schizophrenia patients yield a higher prediction error in schizophrenia subjects compared to controls. The abnormalities in the response network in schizophrenia, in turn, account for the cognitive deficits in this disorder.

Additionally, for controls, it was found a statistically significant relationship between the amount of change from pre-stimulus to cognitive response and the model that better predicts the cognitive network. Fig. 5B shows that the secondary pathway reinforcement modeling is linked to a higher network reconfiguration in controls, which could be considered the 'normal behavior'. However, schizophrenia patients did not show that trend. This was observed in the theta band, which supports the concept of the impaired top-down processing in schizophrenia (Uhlhaas et al., 2008). This lack of activation of the connections with low synchronization during prestimulus in several schizophrenia patients could be due to several reasons. Thereby, it could be related with abnormal structural connectivity networks (Gomez-Pilar et al., 2018a), with hyperactive functional connectivity in the patients during the pre-stimulus (Gomez-Pilar et al., 2018b), or with deficits in the inhibitory/excitatory circuits, usually linked to glutamate neurotransmission (Moghaddam, 2003), which could elicit abnormalities in the synchronization between brain regions.

Glutamatergic abnormalities could not only be related to the longrange synchronization in the theta band, but also to the way of organizing the connections during a cognitive task. While subjects are waiting for the next relevant stimulus, the brain is in a state of alert related to glutamate resting-state concentration in the perigenual anterior cingulate cortex (PACC) (Bai et al., 2015). This level of glutamatergic activity has a strong relationship with the pre-stimulus oscillations. Therefore, possible abnormalities of glutamatergic concentrations in schizophrenia would affect to the predisposition to change of the pre-stimulus network (Bai et al., 2015). Furthermore, a special association between pre-stimulus activity levels and stimulus-induced activity has been suggested in previous studies (Bai et al., 2015). We can speculate that the aberrant network dynamics during cognition in schizophrenia may be driven by underlying abnormalities in the glutamate resting-state concentration in the PACC. Could these abnormalities impact on the cognitive network model of schizophrenia patients? Could it be the reason for the almost dichotomous distribution of the selected models in the schizophrenia group?

A plausible hypothesis for explaining the heterogeneity of the selected models could be the extended concept that schizophrenia is a complex and heterogeneous disorder with distinguishable genotypes (Sheffield and Barch, 2016) and network abnormalities (Gomez-Pilar et al., 2018a), which can influence the cognitive traits. Schizophrenia heterogeneity should not be related to the symptoms, but to underlying neural mechanisms, which are maybe phase-related. To address this heterogeneity, we measured the correlation between network topology features and the cognitive variables.

\subsection{Relationship between topological network measures and cognitive variables}

Our results showed a positive correlation between the modulation (i.e., change from pre-stimulus to cognitive response) of the connectivity strength and three cognitive domains measured by means of the BACS: processing speed, verbal fluency and verbal memory. Consequently, the higher averaged values of change in synchronization between brain regions would involve a better performance in the measured cognitive traits. Due to the novelty of our approach, we only have notice of one study that previously assessed the association between the modulation of network parameters and cognitive data in schizophrenia (Gomez-Pilar et al., 2017). Despite the difficulty to compare the correlations observed in this study with previous findings and for the sake of brevity, we link the present findings with previous works in the Supplementary material. 


\subsection{Further steps towards a comprehensive neural cognitive model}

Despite the clues provided in this study to obtain a reliable model of the brain network dynamics during cognition, a number of questions remain unresolved. First, brain functioning is complex; probably it is more complex than the proposed model. It could result in losing relevant information about brain interactions likely related to the heterogeneity among subjects. For instance, complementary processes could be taking place, such as changes in specific connections or deactivations of a few well-connected pathways during pre-stimulus. Therefore, an almost infinite number of combinations of changes in synchronization could be analyzed to individually improve the model adjustment, but the generalization capability of the model would be likely lost.

Second, the present study was focused on the theta frequency band. Knowing the importance of other frequency bands in the brain functioning, we focused on theta band because of its close relationship with memory processes, top-down control and long-range interactions in the brain, all of them involved in core features of the schizophrenia pathology. Additionally, it was not feasible to simultaneously determine the model for all the frequency bands due to the high computational cost for individually adjusting the more confident model. Future works should investigate network dynamics in other frequency bands to ascertain the predisposition to change of the pre-stimulus activity.

Finally, a hierarchical clustering analysis using both the graph measures and the cognitive/behavioral data could be useful to check the schizophrenia subgroups found by the proposed EEG model. However, a large number of observations would be needed for this kind of analysis, being insufficient the number of subjects of the present study. Future studies with larger number of subjects should address this issue.

\section{Conclusions}

We provided direct evidence of the predictive capability of the proposed model to ascertain the functional brain behavior during cognition. Our results support the idea that schizophrenia is associated with significant abnormalities in the relation between neural dynamics during the pre-stimulus and cognitive response, which are directly related to cognitive performance. Furthermore, we presented a new model of network organization during cognition based on graph theory measures, which could be used to differentiate behavioral phenotypes of schizophrenia. Our findings not only contribute to a further understanding of healthy neural dynamics during cognition, but also provide new insights for identifying the altered neural underpinnings of schizophrenia.

\section{Role of the funding source}

This research project was supported in part by "Ministerio de Economía y Competitividad” and FEDER (TEC2014-53196-R), by “European Commission' (POCTEP 0378_AD_EEGWA_2_P), by “Consejería de Educación de la Junta de Castilla y León’ (VA037U16), by "Fondo de Investigaciones Sanitarias (Instituto de Salud Carlos III)" under projects FIS PI11/02203 and PI15/00299, and by “Gerencia Regional de Salud de Castilla y León” under projects GRS 932/A/14 and GRS 1134/A/15. A. Lubeiro was in receipt of a grant from the Consejería de Educación (Junta de Castilla y León). J. Gomez-Pilar was in receipt of a grant from University of Valladolid.

\section{Contributors}

J. Poza, R. Hornero and G. Northoff designed the study.J. Gomez-Pilar, C. Gómez, J. Poza and R. Hornero analyzed the EEGs and undertook the statistical analyses. J. Gomez-Pilar wrote the manuscript and performed the computational modeling. V. Molina recruited the patients and performed clinical assessment. A. Lubeiro and B. Cea-Cañas performed the electroencephalographic recordings and the cognitive assessment. All authors contributed to the article, revised and approved the final manuscript.

\section{Conflict of interest}

The authors report no biomedical financial interests or potential conflicts of interest.

\section{Acknowledgement}

The authors thank Mr. Martinez-Cagigal for providing the Matlab scripts that significantly enhance the appearance of some figures of the manuscript. We also would like to express our gratitude to the Psychiatry Service of the Clinical University Hospital of Valladolid (Spain), for their help and support and the altruistic volunteers for participation.

\section{Appendix A. Supplementary data}

Supplementary data to this article can be found online at https://doi. org/10.1016/j.schres.2018.04.043.

\section{References}

American Psychiatric Association, 2013. Diagnostic and Statistical Manual of Mental Disorders. 5th Edition. American Psychiatric Publishing, Arlington DSM-5. https://doi. org/10.1176/appi.books.9780890425596.893619.

Bachiller, A., Díez, A., Suazo, V., Domínguez, C., Ayuso, M., Hornero, R., Poza, J., Molina, V., 2014. Decreased spectral entropy modulation in patients with schizophrenia during a P300 task. Eur. Arch. Psychiatry Clin. Neurosci. 264:533-543. https://doi.org/10.1007/ s00406-014-0488-6.

Bachiller, A., Poza, J., Gómez, C., Molina, V., Suazo, V., Hornero, R., 2015. A comparative study of event-related coupling patterns during an auditory oddball task in schizophrenia. J. Neural Eng. 12:16007. https://doi.org/10.1088/1741-2560/12/1/016007.

Bai, Y., Nakao, T., Xu, J., Qin, P., Chaves, P., Heinzel, A., Duncan, N., Lane, T., Yen, N.-S., Tsai, S.-Y., Northoff, G., 2015. Resting state glutamate predicts elevated pre-stimulus alpha during self-relatedness: a combined EEG-MRS study on "rest-self overlap". Soc. Neurosci. 919:1-15. https://doi.org/10.1080/17470919.2015.1072582.

Barch, D.M., Sheffield, J.M., 2014. Cognitive impairments in psychotic disorders: common mechanisms and measurement. World Psychiatry 13:224-232. https://doi.org/ 10.1002/wps.20145.

Bassett, D.S., Bullmore, E., 2006. Small-world brain networks. Neuroscientist 12:512-523. https://doi.org/10.1177/1073858406293182.

Bastos, A.M., Schoffelen, J.-M., 2016. A tutorial review of functional connectivity analysis methods and their interpretational pitfalls. Front. Syst. Neurosci. 9:175. https://doi. org/10.3389/fnsys.2015.00175.

Bjorkquist, O.A., Olsen, E.K., Nelson, B.D., Herbener, E.S., 2016. Altered amygdala-prefrontal connectivity during emotion perception in schizophrenia. Schizophr. Res. 175: 35-41. https://doi.org/10.1016/j.schres.2016.04.003.

Bob, P., Palus, M., Susta, M., Glaslova, K., 2008. EEG phase synchronization in patients with paranoid schizophrenia. Neurosci. Lett. 447:73-77. https://doi.org/10.1016/j. neulet.2008.09.055.

Boccaletti, S., Latora, V., Moreno, Y., Chavez, M., Hwang, D.U., 2006. Complex networks: structure and dynamics. Phys. Rep. 424:175-308. https://doi.org/10.1016/j. physrep.2005.10.009.

Buzsáki, G., 2005. Theta rhythm of navigation: link between path integration and landmark navigation, episodic and semantic memory. Hippocampus 15:827-840. https://doi.org/10.1002/hipo.20113.

Clare, L., 1993. Memory in schizophrenia: what is impaired an what is perserved? Neuropsychologia 31, 1225-1241.

Deco, G., Tononi, G., Boly, M., Kringelbach, M.L., 2015. Rethinking segregation and integration: contributions of whole-brain modelling. Nat. Rev. Neurosci. 16:430-439. https://doi.org/10.1038/nrn3963.

Dickerson, D.D., Wolff, A.R., Bilkey, D.K., 2010. Abnormal long-range neural synchrony in a maternal immune activation animal model of schizophrenia. J. Neurosci. 30: 12424-12431. https://doi.org/10.1523/JNEUROSCI.3046-10.2010.

Ford, J.M., Mathalon, D.H., Whitfield, S., Faustman, W.O., Roth, W.T., 2002. Reduced communication between frontal and temporal lobes during talking in schizophrenia. Biol. Psychiatry 51:485-492. https://doi.org/10.1016/S0006-3223(01)01335-X.

Friston, K.J., 1998. The disconnection hypothesis. Schizophr. Res. 30:115-125. https://doi. org/10.1016/S0920-9964(97)00140-0.

Friston, K., Frilh, C.D., 1995. Schizophrenia: a disconnection syndrome? Clin. Neurosci. 3, 89-97.

Friston, K., Kiebel, S., 2009. Predictive coding under the free-energy principle. Philos. Trans. R. Soc. B Biol. Sci. 364:1211-1221. https://doi.org/10.1098/rstb.2008.0300.

Friston, K.J., Harrison, L., Penny, W., 2003. Dynamic causal modelling. NeuroImage 19: 1273-1302. https://doi.org/10.1016/S1053-8119(03)00202-7.

Friston, K., Brown, H.R., Siemerkus, J., Stephan, K.E., 2016. The dysconnection hypothesis (2016). Schizophr. Res. 176:83-94. https://doi.org/10.1016/j.schres.2016.07.014.

Gomez-Pilar, J., Poza, J., Bachiller, A., Gómez, C., Molina, V., Hornero, R., 2015. Neural network reorganization analysis during an auditory oddball task in schizophrenia using wavelet entropy. Entropy 17:5241-5256. https://doi.org/10.3390/e17085241.

Gomez-Pilar, J., Lubeiro, A., Poza, J., Hornero, R., Ayuso, M., Valcárcel, C., Haidar, K., Blanco, J.A., Molina, V., 2017. Functional EEG network analysis in schizophrenia: evidence of larger segregation and deficit of modulation. Prog. Neuro-Psychopharmacol. Biol. Psychiatry 76:116-123. https://doi.org/10.1016/j.pnpbp.2017.03.004.

Gomez-Pilar, J., de Luis-García, R., Lubeiro, A., de la Red, H., Poza, J., Núñez, P., Hornero, R. Molina, V., 2018a. Relations between structural and EEG-based graph metrics in healthy controls and schizophrenia patients. Hum. Brain Mapp. https://doi.org/ 10.1002/hbm.24066.

Gomez-Pilar, J., de Luis-García, R., Lubeiro, A., de Uribe, N., Poza, J., Núñez, P., Ayuso, M., Hornero, R., Molina, V., 2018b. Deficits of entropy modulation in schizophrenia are predicted by functional connectivity strength in the theta band and structural clustering. NeuroImage Clin. 18:382-389. https://doi.org/10.1016/j.nicl.2018.02.005.

Gomez-Pilar, J., Poza, J., Bachiller, A., Gómez, C., Núñez, P., Lubeiro, A., Molina, V., Hornero, R., 2018c. Quantification of graph complexity based on the edge weight distribution balance: application to brain networks. Int. J. Neural Syst. 28:1750032. https://doi. org/10.1142/S0129065717500320.

Hohwy, J., Roepstorff, A., Friston, K., 2008. Predictive coding explains binocular rivalry: an epistemological review. Cognition 108:687-701. https://doi.org/10.1016/j. cognition.2008.05.010. 
Kapur, S., 2003. Psychosis as a state of aberrant salience: a framework linking biology, phenomenology, and pharmacology in schizophrenia. Am. J. Psychiatry 160:13-23. https://doi.org/10.1176/appi.ajp.160.1.13.

Kikuchi, M., Koenig, T., Wada, Y., Higashima, M., 2007. Native EEG and Treatment Effects in Neuroleptic-naïve Schizophrenic patients: Time and Frequency Domain Approaches. 97:pp. 163-172. https://doi.org/10.1016/j.schres.2007.07.012.

Kilner, J.M., Friston, K.J., Frith, C.D., 2007. Predictive coding: an account of the mirror neuron system. Cogn. Process. 8:159-166. https://doi.org/10.1007/s10339-007-0170-2.

Kim, J.-J., Kwon, J.S., Park, H.J., Youn, T., Kang, D.H., Kim, M.S., Lee, D.S., Lee, M.C., 2003. Functional disconnection between the prefrontal and parietal cortices during working memory processing in schizophrenia: a [150]H2O PET study. Am. J. Psychiatry 160:919-923. https://doi.org/10.1176/appi.ajp.160.5.919.

Koenig, T., Lehmann, D., Saito, N., Kuginuki, T., Kinoshita, T., Koukkou, M., 2001. Decreased functional connectivity of EEG theta-frequency activity naïve patients with schizophrenia: preliminary results. Schizophr. Res. 50, 55-60.

Lakatos, P., Musacchia, G., O'Connel, M., Falchier, A., Javitt, D., Schroeder, C., 2013. The spectrotemporal filter mechanism of auditory selective attention. Neuron 77: 750-761. https://doi.org/10.1016/j.neuron.2012.11.034.

Leech, R., Sharp, D.J., 2014. The role of the posterior cingulate cortex in cognition and disease. Brain 137:12-32. https://doi.org/10.1093/brain/awt162.

Lewis, D.A., Hashimoto, T., Volk, D.W., 2005. Cortical inhibitory neurons and schizophrenia. Nat. Rev. Neurosci. 6:312-324. https://doi.org/10.1038/nrn1648.

Li, F., Chen, B., Li, H., Zhang, T., Wang, F., Jiang, Y., Li, P., Ma, T., Zhang, R., Tian, Y., Liu, T. Guo, D., Yao, D., Xu, P., 2016. The time-varying networks in P300: a task-evoked EEG study. IEEE Trans. Neural Syst. Rehabil. Eng. 24:725-733. https://doi.org/ 10.1109/TNSRE.2016.2523678.

Liu, Y., Liang, M., Zhou, Y., He, Y., Hao, Y., Song, M., Yu, C., Liu, H., Liu, Z., Jiang, T., 2008 Disrupted small-world networks in schizophrenia. Brain 131:945-961. https://doi. org/10.1093/brain/awn018.

Ma, S., Calhoun, V.D., Eichele, T., Du, W., Adali, T., 2012. Modulations of functional connectivity in the healthy and schizophrenia groups during task and rest. Neurolmage 62 1694-1704. https://doi.org/10.1016/j.neuroimage.2012.05.048.

Micheloyannis, S., Pachou, E., Stam, C.J., Breakspear, M., Bitsios, P., Vourkas, M., Erimaki, S Zervakis, M., 2006. Small-world networks and disturbed functional connectivity in schizophrenia. Schizophr. Res. 87:60-66. https://doi.org/10.1016/j.schres.2006.06.028.

Moghaddam, B., 2003. Bringing order to the glutamate chaos in schizophrenia. Neuron 40:881-884. https://doi.org/10.1016/S0896-6273(03)00757-8.

Moustafa, A.A., Gluck, M.A., 2011. Computational cognitive models of prefrontal-striatalhippocampal interactions in Parkinson's disease and schizophrenia. Neural Netw. 24:575-591. https://doi.org/10.1016/j.neunet.2011.02.006.

Mukherjee, P., Sabharwal, A., Kotov, R., Szekely, A., Parsey, R., Barch, D.M., Mohanty, A., 2016. Disconnection between amygdala and medial prefrontal cortex in psychotic disorders. Schizophr. Bull. 42:1056-1067. https://doi.org/10.1093/schbul/sbw012.

Northoff, G., Duncan, N.W., 2016. How do abnormalities in the brain's spontaneous activity translate into symptoms in schizophrenia? From an overview of resting state activity findings to a proposed spatiotemporal psychopathology. Prog. Neurobiol. 145146:26-45. https://doi.org/10.1016/j.pneurobio.2016.08.003.

Poppe, A.B., Barch, D.M., Carter, C.S., Gold, J.M., Ragland, J.D., Silverstein, S.M., MacDonald, A.W., 2016. Reduced frontoparietal activity in schizophrenia is linked to a specific deficit in goal maintenance: a multisite functional imaging study. Schizophr. Bull. 42:1149-1157. https://doi.org/10.1093/schbul/sbw036.

Power, J.D., Schlaggar, B.L., Lessov-schlaggar, C.N., Petersen, S.E., 2013. Article evidence for hubs in human functional brain networks. Neuron 79:798-813. https://doi.org/ 10.1016/j.neuron.2013.07.035.

Reijneveld, J.C., 2011. Altered small-world brain networks in temporal lobe in patients with schizophrenia performing an auditory oddball task. Front. Syst. Neurosci. 5: 1-13. https://doi.org/10.3389/fnsys.2011.00007.

Rossell, S., Batty, R., 2008. Elucidating semantic disorganisation from a word comprehension task: do patients with schizophrenia and bipolar disorder show differential processing of nouns, verbs and adjectives? Schizophr. Res. 102:63-68. https://doi.org/ 10.1016/j.schres.2008.04.008.

Rubinov, M., Sporns, O., 2010. Complex network measures of brain connectivity: uses and interpretations. NeuroImage 52:1059-1069. https://doi.org/10.1016/j. neuroimage.2009.10.003.
Sánchez-Morla, E.M., Barabash, A., Martínez-Vizcaíno, V., Tabarés-Seisdedos, R., BalanzáMartínez, V., Cabranes-Díaz, J.A., Baca-Baldomero, E., Gómez, J.L.S., 2009. Comparative study of neurocognitive function in euthymic bipolar patients and stabilized schizophrenic patients. Psychiatry Res. 169:220-228. https://doi.org/10.1016/j. psychres.2008.06.032

Segarra, N., Bernardo, M., Gutierrez, F., Justicia, A., Fernadez-Egea, E., Allas, M., Safont, G., Contreras, F., Gascon, J., Soler-Insa, P.A., Menchon, J.M., Junque, C., Keefe, R.S.E., 2011. Spanish validation of the Brief Assessment in Cognition in Schizophrenia (BACS) in patients with schizophrenia and healthy controls. Eur. Psychiatry 26: 69-73. https://doi.org/10.1016/j.eurpsy.2009.11.001.

Sheffield, J.M., Barch, D.M., 2016. Cognition and resting-state functional connectivity in schizophrenia. Neurosci. Biobehav. Rev. 61:108-120. https://doi.org/10.1016/j. neubiorev.2015.12.007.

Shim, M., Kim, D.W., Lee, S.H., Im, C.H., 2014. Disruptions in small-world cortical functional connectivity network during an auditory oddball paradigm task in patients with schizophrenia. Schizophr. Res. 156:197-203. https://doi.org/10.1016/j. schres.2014.04.012.

Sigurdsson, T., Stark, K.L., Karayiorgou, M., Gogos, J.A., Gordon, J.A., 2010. Impaired hippocampal - prefrontal synchrony in a genetic mouse model of schizophrenia. Nature 464:763-767. https://doi.org/10.1038/nature08855.

Simonsen, C., Sundet, K., Vaskinn, A., Birkenaes, A.B., Engh, J.A., Faerden, A., Jonsdottir, H., Ringen, P.A., Opjordsmoen, S., Melle, I., Friis, S., Andreassen, O.A., 2011. Neurocognitive dysfunction in bipolar and schizophrenia spectrum disorders depends on history of psychosis rather than diagnostic group. Schizophr. Bull. 37: 73-83. https://doi.org/10.1093/schbul/sbp034.

Thai, N.J., Longe, O., Rippon, G., 2009. Disconnected brains: what is the role of fMRI in connectivity research? Int. J. Psychophysiol. 73:27-32. https://doi.org/10.1016/j. ijpsycho.2008.12.015.

Tononi, G., Edelman, G.M., Sporns, O., 1998. Complexity and coherency: integrating information in the brain. Trends Cogn. Sci. 2, 474-484.

Uhlhaas, P.J., Singer, W., 2010. Abnormal neural oscillations and synchrony in schizophrenia. Nat. Rev. Neurosci. 11:100-113. https://doi.org/10.1038/nrn2774.

Uhlhaas, P.J., Haenschel, C., Nikolić, D., Singer, W., 2008. The role of oscillations and synchrony in cortical networks and their putative relevance for the pathophysiology of schizophrenia. Schizophr. Bull. 34:927-943. https://doi.org/10.1093/schbul/sbn062.

van den Heuvel, M.P., Fornito, A., 2014. Brain networks in schizophrenia. Neuropsychol. Rev. 24:32-48. https://doi.org/10.1007/s11065-014-9248-7.

Varela, F., Lachaux, J.P., Rodriguez, E., Martinerie, J., 2001. The brainweb: phase synchronization and large-scale integration. Nat. Rev. Neurosci. 2:229-239. https://doi.org/ $10.1038 / 35067550$.

Vöhringer, P.A., Barroilhet, S.A., Amerio, A., Reale, M.L., Alvear, K., Vergne, D., Ghaemi, S.N., 2013. Cognitive impairment in bipolar disorder and schizophrenia: a systematic review. Front. Psychiatry 4:1-11. https://doi.org/10.3389/fpsyt.2013.00087.

Whalley, H.C., 2005. Functional disconnectivity in subjects at high genetic risk of schizophrenia. Brain 128:2097-2108. https://doi.org/10.1093/brain/awh556.

Xia, M., Wang, J., He, Y., 2013. BrainNet viewer: a network visualization tool for human brain connectomics. PLoS One 8, e68910. https://doi.org/10.1371/journal. pone.0068910.

Yeo, R.A., Ryman, S.G., van den Heuvel, M.P., de Reus, M.A., Jung, R.E., Pommy, J., Mayer, A. R., Ehrlich, S., Schulz, S.C., Morrow, E.M., Manoach, D., Ho, B., Sponheim, S.R., Calhoun, V.D., 2016. Graph metrics of structural brain networks in individuals with schizophrenia and healthy controls: group differences, relationships with intelligence, and genetics. J. Int. Neuropsychol. Soc. 22:240-249. https://doi.org/10.1017/ S1355617715000867.

Zanelli, J., Reichenberg, A., Morgan, K., Fearon, P., Kravariti, E., Dazzan, P., Morgan, C., Zanelli, C., Demjaha, A., Jones, P.B., Doody, G.A., Kapur, S., Murray, R.M., 2010. Specific and generalized neuropsychological deficits: comparison of patients with various first-episode psychosis presentations. Am. J. Psychiatry 167:78-85. https://doi.org/ 10.1176/appi.ajp.2009.09010118. 